\title{
La cultura escolar en el contexto de la inserción social adolescente
}

\section{Dora Herrera}

Universidad de Lima

Lima, Perú

En el contexto de un proyecto mayor orientado a conocer los determinantes psicológicos y sociales de las estrategias de inserción social que utilizan los adolescentes que culminan la educación secundaria, se ha realizado la evaluación de la cultura escolar en 321 sujetos de cinco centros educativos estatales mixtos de los distritos de Pamplona Alta, Miraflores y San Isidro. Para desarrollar el presente estudio se diseñó y aplicó una escala tipo Likert que evaluó la cultura escolar orientada hacia las áreas de educación postsecundaria, empleo y vida familiar. Los resultados han mostrado diferencias significativas en la cultura escolar denominada A (alta orientación a la inserción social) y B (baja o implícita orientación a la inserción social).

cultura escolar/ inserción social/ tareas de desarrollo

\section{School culture in the context of adolescent social insertion}

School culture assessment has been applied in 321 subjets who belong to five public schools in Pamplona Alta and Miraflores. This evaluation has been developed in the context of a larger project oriented to understand the social and psychological determinants of social insertion strategies in adolescents who have finished high school level. To develop this study a Likert type scale has been designed and applied. The scale considered three main areas: post secondary education, employment, and family life. The results have pointed out statistical significant differences between the school culture type A (high or explicit orientation to social insertion) and the school culture type B (low or implicit orientation to social insertion). 


\section{INTRODUCCIÓN}

La expresión del crecimiento y desarrollo de los países latinoamericanos, y específicamente del Perú, no se sustenta exclusivamente en indicadores económicos; el desarrollo socioeconómico se relaciona también con el bienestar de la población, pues existe una dimensión humana y psicológica que puede observarse a través de otros indicadores sociales, entre los que destacan los de pobreza, distribución de ingresos, empleo, calidad y cobertura de los servicios públicos (Parodi, 1997).

Tomando en consideración los aspectos sociales y psicológicos, que son parte del desarrollo nacional, se realizó el presente estudio que tiene como propósito conocer con precisión cuáles son las estrategias de inserción social que emplean los jóvenes luego de concluir sus estudios secundarios.

Para llevarlo a cabo se revisó inicialmente información reportada por el Instituto Nacional de Estadística e Informática (INEI, 1998) según la cual es posible concluir que el Perú contará en los próximos años con la mayor generación de jóvenes de su historia, lo cual creará, de manera inevitable, un aumento de la población en edad activa.

Esta situación poblacional nos brinda una gran oportunidad para el desarrollo de nuestro país, pero debe existir una adecuada comprensión de las características psicológicas de nuestros recursos humanos, pues no bastan las inversiones sociales en salud, educación y empleo para que esto revierta de manera contundente en la mejora de la calidad de vida.

En primer término se revisan algunas cifras que ponen en evidencia particularidades de la situación de empleo y desempleo juvenil en el Perú.

De acuerdo con las normas internacionales, la población de catorce años de edad o más, es la que se encuentra apta para trabajar. En el Perú, en el ámbito urbano, la Población Económicamente Activa (PEA) está constituida en $91,7 \%$ por los que tienen un empleo o se encuentran ocupados y en $8,3 \%$ por los que buscan un empleo u ocupación (INEI, 1997).

Los reportes de los años anteriores emitidos por el INEI (1995-1996) indican que el porcentaje de mujeres que constituyen la PEA alcanza el 40,6\% y 41,2\%, respectivamente. En 1997 el desempleo urbano afecta más a la PEA femenina

El desempleo o desocupación ha venido siendo considerado como un problema significativo en el país, en especial para el sector juvenil y femenino de nuestra población.

Según algunos estudios recientes (UNICEF-Cuánto, 1997), el desempleo en las áreas urbanas afecta al $20 \%$ de la población femenina y al $17,4 \%$ de la juvenil (cuyas edades oscilan entre 14 y 24 años). Es importante señalar también el incremento anual del porcentaje de la PEA con estudios secundarios, pues éste se aumentó de 43,5\% a 
44,8\% en el período de un año (19951996) (INEI, 1997).

PEA-Nivel educativo \%



Resulta también importante resaltar que la mayor parte de la PEA se concentra en Lima Metropolitana.

PEA-Ámbito geográfico \%

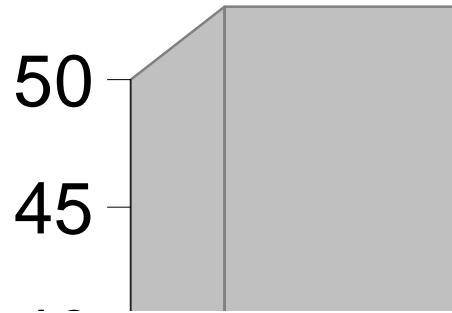

$\mathrm{Al}$ analizar la composición de la PEA por grupos de edad a lo largo de un período, es posible inferir que la mayor proporción en la estructura de ésta -cerca de la mitad (48,6\%)- durante 1997 tenía entre 25 y 44 años, sin embargo, las cifras correspondientes al grupo que se encuentra entre 14 y 24 años también deben ser consideradas, pues en 1997 alcanzaron el 26,3\%.

\section{PEA-Grupos de edad \%}



La inserción en el mercado de trabajo y la consecución de un empleo entre aquellos que no poseen un elevado nivel de educación es más difícil. Es ilustrativa la información que se reporta con respecto a quienes sólo poseen estudios secundarios $(52,1 \%$ en 1997$)$ (INEI, 1998).

Sin bien el desempleo es un problema que afecta de manera significativa a los jóvenes en nuestro país, la dificultad real es la del subempleo. Un estudio reciente publicado por UNICEF y el Instituto Cuánto precisó que el $90 \%$ de la PEA se encuentra "trabajando" pero su salario es muy bajo y no poseen seguridad de permanecer en dicho empleo. 
De acuerdo con lo mencionado, es claro que la población juvenil se encuentra en una situación crítica. La necesidad de insertarse en el mercado laboral de parte de los jóvenes, aunada a su pobre nivel de calificación para hacerlo, los ubica en el grupo de los subempleados, y es por ello que inician su actividad laboral como "empleadas del hogar" (9,2\%), asistentes de almacenes $(9,1 \%)$, ambulantes $(8,2 \%)$ o como colaboradores en el área de transporte $(6,4 \%)$. Otros no poseen una categoría clara en la cual ubicarlos (Ministerio de Trabajo, 1997).

Además de las cifras descritas por el INEI, que ilustran la situación de los jóvenes en nuestro país, y los estudios previos realizados en la zona de Pamplona, resulta relevante considerar como un elemento adicional en el contexto juvenil el hecho de que el Ministerio de Trabajo, mediante el proyecto denominado Pro-Joven, analizó los obstáculos que los jóvenes peruanos tienen para insertarse en el mercado de trabajo.

Se ha considerado que una de las principales dificultades es no poseer experiencia laboral. A través del mencionado proyecto, actualmente se brinda soporte a los jóvenes para que se capaciten en empresas que, mediante un acuerdo previo con el Ministerio, los entrenan por un período corto y de manera eficiente. Los resultados iniciales han sido favorables para los jóvenes inicialmente reclutados.

\section{AnTECEDENTES}

\section{Primer estudio exploratorio en Pamplona Alta}

Desde una perspectiva psicosocial resultó importante conocer la percepción que con respecto a los problemas sociales poseían los pobladores de un sector pauperizado de Lima, específicamente Pamplona Alta.

Para ello, se aplicó una encuesta y una entrevista estructurada a las mujeres representantes de los comedores populares que se encontraban organizadas en los diferentes pueblos jóvenes de la zona mencionada. Se evaluó a 79 mujeres que pertenecían a 14 comedores populares de la zona y los resultados obtenidos permitieron precisar un conjunto de características sociodemográficas, pero, sobre todo, mediante la entrevista fue posible obtener información en torno a los problemas percibidos por ellas.

El análisis de la información reportada se encuentra interrelacionada; sin embargo, ésta fue jerarquizada llegándose a señalar que en primera instancia debía mejorarse los servicios públicos de la zona, específicamente el referido a la recolección de basura.

Como segundo problema percibido se identificó la violencia juvenil, la cual se había incrementado notablemente en los pueblos jóvenes con las pandillas presentes de manera permanente. 
Otros problemas mencionados también fueron los referidos al elevado consumo de drogas y a la limitada seguridad ciudadana.

Finalmente se hizo referencia a las consecuencias de la pobreza, tales como la limitada canasta familiar, las dificultades económicas para acceder a los servicios de salud y el transporte.

\section{Segundo estudio en Pamplona Alta}

$\mathrm{Al}$ conocer los problemas reportados por las mujeres entrevistadas en el primer estudio, se consideró conveniente desarrollar una propuesta de trabajo comunitario para cada uno de ellos. Se descartó la mejora de los servicios públicos ya que requería de un trabajo multidisciplinario y de organización social que no podía ser desarrollado en términos inmediatos.

Con respecto al segundo problema, el de la violencia juvenil e incremento de pandillas, se buscó información más específica sobre la realidad social de los jóvenes y se visitó a los estudiantes de uno de los centros educativos más reconocidos de Pamplona (San Luis Gonzaga).

Se solicitó a los estudiantes de tercero, cuarto y quinto de secundaria que identifiquen, en orden de importancia, los problemas sociales de su comunidad. El resultado que se obtuvo fue el siguiente:

- violencia juvenil (pandillas),

- incomprensión de los padres,

- violencia general, e
- inadecuados servicios públicos.

Se indagó también acerca de sus expectativas e intereses y se obtuvo la siguiente información:

- necesidad de tener una profesión,

- conseguir empleo,

- tener una familia que ayude a solucionar los problemas personales, y

- lograr una vida confortable.

Los datos obtenidos a través de los estudios en Pamplona nos orientaron para la realización de un nuevo análisis que será descrito a continuación.

\section{ESTUDIO PILOTO PARA CONOCER LOS}

PLANES Y ACTIVIDADES DE LOS

\section{EGRESADOS DE SECUNDARIA}

$\mathrm{Al}$ analizar los reportes de las mujeres que participaban de los comedores populares y de los jóvenes escolares, se observó una notable coincidencia en indicar que uno de los problemas que enfrentaba la comunidad era el de la violencia juvenil.

Ante esta evidencia se organizó un estudio piloto con el fin de conocer cuáles son los planes que tienen los jóvenes que han concluido sus estudios secundarios. Asimismo se reportaron cuáles eran sus principales actividades cotidianas.

A través de una entrevista estructurada se visitó a 75 jóvenes que habían concluido sus estudios en el Colegio San Luis Gonzaga. Se recolectó información acerca de los planes que poseían antes de culminar sus estudios secundarios. Los resultados permitieron 
establecer que el $60 \%$ de ellos había planeado estudiar en la universidad y $30 \%$ en instituciones técnicas, especialmente porque consideraban que ingresar a la universidad iba a resultar muy difícil. Sólo el 3\% quería conseguir un trabajo y el $7 \%$ restante esperaba trabajar mientras estudiaba.

A pesar de que las expectativas estudiantiles eran muy altas con respecto a la universidad, sólo el $9 \%$ de ellos postuló. El 33\% estaba trabajando mientras $15 \%$ se encontraba estudiando en instituciones técnicas. El 9\% estudiaba en academias preuniversitarias y el $43 \%$ de la población entrevistada no realizaba ninguna actividad específica $y$ se encontraba generalmente en casa durante el día. El porcentaje de jóvenes que no realiza ninguna actividad específica resulta relevante para presentar una nueva propuesta de investigación más sistemática, con algunas interrogantes que se agreguen a las planteadas durante el trabajo realizado en Pamplona Alta, como las que se precisan a continuación.

- ¿Cuáles son los determinantes sociales e individuales de la inserción social en estudiantes que han culminado sus estudios secundarios y que provienen de diferentes estratos socioeconómicos?

- ¿Existen diferencias en la inserción social de los estudiantes que provienen de Centros Educativos con una cultura escolar distinta?

- ¿Cómo perciben los profesores y padres de los estudiantes las normas o criterios que se usan en su entorno social para considerar que un estudiante ha sido exitoso al integrarse en la sociedad?

- ¿Existen diferencias significativas entre los niveles socioeconómicos con respecto a las expectativas de padres y maestros de los egresados de la escuela secundaria?

- ¿Es distinta la formulación de los proyectos de vida individual con respecto a la educación futura, ocupacional y familiar de los egresados de la escuela secundaria que provienen de distintos niveles socioeconómicos.

- ¿Cómo se insertan en el ámbito educacional, profesional y social los estudiantes de dos estratos socioeconómicos?

\section{JUSTIFICACIÓN}

\section{Contexto sociocultural, educación e inserción social}

En el campo de la psicología social del desarrollo se han realizado estudios que indican que la participación educativa y las aspiraciones de los adolescentes se encuentran asociadas a aspectos relevantes de sus familias y entorno escolar.

Los estudiantes que poseen familias cognitivamente estimulantes, estables, afectuosas y solventes, tienen ciertas ventajas con respecto a su participación en el sistema educativo; tienden a 
permanecer en dicho sistema por períodos más prolongados y sus aspiraciones son diferentes a las de los adolescentes que provienen de familias ubicadas en el nivel socioeconómico bajo, pues estos últimos tienden a aspirar a ocupaciones de menor calificación (Durkin, 1996).

Otras investigaciones han reportado que existe una relación estrecha entre el nivel educativo y las posibilidades de lograr mayores ingresos económicos. Se considera que a mayor período de permanencia en el sistema educativo es más alta la probabilidad de lograr mejores posiciones laborales y de obtener mayores ingresos (Psacharopoulos, 1994).

De acuerdo con los reportes de investigación antes mencionados es posible señalar que la educación no sólo es un elemento clave en el desarrollo social, sino que le brinda a los individuos la posibilidad de incrementar sus capacidades y habilidades de modo que posteriormente éstos puedan realizar mejores elecciones (Parodi, 1997).

La educación formal generalmente se ofrece en el ámbito público y privado. La calidad educativa de estas dos modalidades ha sido comparada en un estudio realizado por Jiménez y otros (1991). Los autores analizaron escuelas secundarias en cinco países en vías de desarrollo: Colombia, Tanzania, Tailandia, Filipinas y República Dominicana. La pregunta central en este trabajo comparativo buscaba conocer si un estudiante seleccionado al azar tenía un mejor rendimiento en la escuela privada que en la pública.

La investigación controló cuidadosamente el entorno de los sujetos, su motivación y habilidades innatas. Los resultados mostraron que los estudiantes de la escuela privada rendían mejor en matemáticas y lenguaje; estos estudiantes aprendían tres veces más que sus pares en la escuela pública.

Se reportó, adicionalmente, que el entorno tiende a ser más favorable para los estudiantes de la escuela privada, pues el ingreso familiar, el nivel educativo de los padres y su situación laboral es diferente y estos aspectos influyen en la elección de la escuela y en el desarrollo de las aspiraciones educativas posteriores de sus hijos.

Los autores del estudio concluyeron, a partir de lo señalado, que los alumnos de las escuelas públicas se encuentran en desventaja, pero asistir a este tipo de escuela es mejor que no poseer ningún tipo de experiencia educativa formal.

Pero no es sólo la familia la relevante en la organización de las opciones de inserción social de los individuos, el contexto sociocultural del adolescente influye también en su desarrollo personal.

Actualmente existe un interés creciente en conocer la forma como dicho contexto influye en el individuo (Lerner, 1998). Los investigadores subrayan la existencia de tareas de desarrollo orientadas de acuerdo con la 
edad, es decir, se considera que existen ciertos caminos estándar que se plantean socialmente según el período de desarrollo en el que se encuentra el individuo.

De acuerdo con Hagestad y Neugarten (1985) "las tareas de desarrollo crean alternativas comportamentales predecibles, expectativas normativas, 'mapas' para la vida", estas tareas aparecen en ciertos períodos de desarrollo de los individuos; si éstos cumplen con ellas se sienten satisfechos y con una sensación de bienestar, si fallan, sucede lo contrario.

La escuela cumple en este proceso también un rol relevante, ésta es considerada generalmente como un sistema humano organizacional que posee su propia cultura. Las constantes observaciones de las instituciones escolares han permitido concluir que las "escuelas difieren unas de otras en el modo de conducir su trabajo y en los efectos que producen en la vida de los alumnos" (Maehr \& Midgley, 1996).

Pese a que la mayoría de las escuelas han sido diseñadas para proveer "un poco de todo para diversos grupos", puede encontrarse diferencias en el énfasis que se pone en algunos contenidos teóricos: el arte, el deporte, la futura vida profesional o laboral, la orientación vocacional, etc.

Las escuelas en general pueden identificar sus prioridades y orientar a los estudiantes con la convicción de que se encuentran en el camino correcto.
En nuestro país la idea de que la escuela es un requisito indispensable en el camino hacia el progreso ha movilizado grandes energías (Ansión et al., 1998).

Las expectativas de los padres con respecto a la escuela y la cultura de ésta serán en el presente estudio variables relevantes.

\section{Tareas de desarrollo y formulación de metas personales}

Los trabajos de Nurmi en Finlandia (1993) articulan las tareas de desarrollo con la formulación de metas personales. En el período juvenil, las metas de los jóvenes se relacionan con las tareas de desarrollo de esa edad. Por ejemplo, se espera que los jóvenes inicien sus actividades laborales, se inserten educativamente, seleccionen una pareja e inicien posteriormente su vida familiar.

Los estudios realizados con jóvenes señalan que éstos mencionan con mayor frecuencia metas relacionadas con su educación futura y vida familiar que las personas mayores. Otras investigaciones reportan también que en una sociedad plural, los subgrupos pueden tener sus propios sistemas de orientación de tareas de desarrollo (Hagestad \& Neugarten, 1985).

Lerner (1998) ha enfatizado el hecho de que son las propias personas las que construyen y producen sus vidas, ellos son los propios agentes de la construcción del futuro y para ello elaboran sus metas personales (Nurmi, 1993). 
La manifestación de dichas metas o su proceso de construcción ha sido descrito mediante diferentes conceptos tales como: metas futuras (Nurmi, 1993); tareas de vida; proyectos personales y striving.

\section{Construcción de metas y perspectiva de tiempo futuro}

En este contexto, desde una aproximación psicológica, la perspectiva de tiempo futuro (PTF) es muy importante; el futuro es un espacio para la cognición humana y el comportamiento; es el espacio para cultivar proyectos de vida (Zaleski, 1994). Los seres humanos, con su capacidad para la representación cognitiva, pueden proyectar sus pensamientos y metas realistas hacia el futuro.

La PTF representa la magnitud y la forma mediante la cual el futuro cronológico es integrado en el espacio del presente. Esta dimensión futura puede ser corta o muy larga, puede incluso extenderse más allá del tiempo de vida individual (Lens, 1998).

La PTF tiene también relevancia motivacional; las diferencias individuales en la extensión de la PTF afectan la intensidad de las metas planteadas en el presente (Lens, 1994). Las personas con una PTF larga son más persistentes en su trabajo por las metas y tienen mayor satisfacción por ello y por las actividades que en el presente se encuentran orientadas hacia dicha meta. No sucede lo mismo con las personas que poseen una PTF más corta (Zaleski, 1994).

Asimismo, la PTF ha sido también asociada con el contexto sociocultural, institucional y familiar de los individuos. Los adolescentes no organizan su perspectiva de tiempo futuro mediante un proceso fundamentalmente intrapsicológico. Éste se encuentra afectado también por la extensión de sus experiencias institucionales y las expectativas de su entorno social. El contexto social en el cual el adolescente madura afecta la construcción de su PTF, el cual sienta las bases cognitivas para la formulación de sus propias metas personales.

Para los jóvenes que egresan de la escuela secundaria el futuro es muy importante. La adolescencia es un período de transición entre la infancia y la adultez. Debido a que los egresados de la escuela secundaria se encuentran en el inicio de su vida adulta independiente, se les permite e incluso alienta permanentemente a pensar en su futuro (Nurmi, 1992).

Algunos estudios señalan que existen diferencias individuales en términos de concebir el futuro y que la eficiencia con la cual un adolescente se prepare para su futuro, reflejará en parte su PTF (Lens, 1994).

De acuerdo con lo anterior es importante resaltar que para los adolescentes o jóvenes la elección de una carrera o empleo es una tarea de desarrollo que depende no sólo de la PTF, sino tam- 
bién, en parte, de cómo conceptúen las personas sus propias habilidades y preferencias.

Las circunstancias socioeconómicas en las que se desarrollan dichos adolescentes condicionan también su orientación hacia el futuro. Muchos países como el Perú, no ofrecen las mejores condiciones de desarrollo individual; especialmente si las personas se encuentran situadas en el sector socioeconómico bajo. Es aquí donde resulta oportuno reflexionar acerca del período de transición hacia el trabajo por el que atraviesa el adolescente luego de culminar la educación secundaria.

La transición tiene beneficios si el joven logra insertarse adecuadamente en la sociedad y luego de apropiadas estrategias consigue un buen empleo. No obstante, si la transición no es exitosa, los jóvenes tienden a mostrar insatisfacción, baja iniciativa y limitada responsabilidad para tomar sus propias decisiones (Durkin, 1996).

\section{VARIABLES DE INVESTIGACIÓN}

El presente estudio es correlacional. Las variables consideradas para realizar la investigación se organizan en tres categorías:

\section{Categoría I}

Entorno social

\section{Nivel socioeconómico}

Se tomó en consideración los criterios del estudio sobre niveles socioeconómicos (NSE) que efectuó
Apoyo Opinión y Mercado S.A. (1998) y los planos estratificados de Lima Metropolitana a nivel de manzanas según estratos socioeconómicos de los hogares que ha elaborado el Instituto Nacional de Estadística.

El presente estudio ha considerado dos grupos el D1 (muy bajo pobre) para el grupo de Pamplona Alta. Y el B2 (medio bajo) para cierto sector poblacional del distrito de Miraflores

\section{Cultura escolar}

La cultura escolar muestra las diferencias en las que pone énfasis la escuela como organización, pudiendo a través de estas particularidades "culturales" orientar a los estudiantes de manera disímil hacia la vida laboral, académica y familiar.

Se diferenciaron dos tipos de cultura: A y B.

La cultura A representa a las escuelas en las que explícitamente se orienta a los estudiantes a la inserción social.

La cultura $B$ representa a las escuelas en las que sólo se orienta a los estudiantes de manera implícita hacia la inserción social o no se les orienta.

\section{Categoría II}

Entorno inmediato:

Expectativas de los padres

Expectativas de los maestros 


\section{Categoría III}

Características personales

Género

Rendimiento escolar

Las variables intermedias

Modelos de rol

Perspectiva de tiempo futuro

La variable dependiente

Inserción social

Movimiento individual a través de un conjunto coherente de experiencias que orienta a los sujetos hacia 'destinos' o espacios sociales identificables.

\section{Muestra}

Se decidió realizar el estudio con los escolares de quinto de secundaria de los centros educativos mixtos y estatales de Pamplona Alta.

El estudio requería un centro educativo con cultura A y otro con cultura B. Se seleccionaron tres centros educativos, dos de cultura B (necesario para incrementar el número de participantes que estudiaban en este tipo de centro) y uno de cultura A.

Para contrastar los resultados con los de otro nivel socioeconómico se procedió en los mismos términos en los centros estatales mixtos de Miraflores y San Isidro.

La muestra para la evaluación de 'cultura escolar' en los centros educativos elegidos quedó finalmente constituida de la siguiente manera:

\begin{tabular}{lccc}
\hline $\begin{array}{l}\text { Centro } \\
\text { educativo }\end{array}$ & $\begin{array}{c}\text { Cultura } \\
\text { A }\end{array}$ & $\begin{array}{c}\text { Cultura } \\
\text { B }\end{array}$ & $\begin{array}{c}\text { Total } \\
\text { (n) }\end{array}$ \\
\hline Ollantay & 75 & & 75 \\
12 de Noviembre & & 28 & 28 \\
San Luis Gonzaga & & 67 & 67 \\
Scipión Llona & & 99 & 99 \\
María Reiche & 52 & & 52 \\
TOTAL (n) & 127 & 194 & 321 \\
\hline
\end{tabular}

\section{EVALUACIÓN DE LA VARIABLE \\ CULTURA ESCOLAR}

Para evaluar 'cultura escolar' se visitaron los centros educativos estatales mixtos Ollantay y San Luis Gonzaga de Pamplona Alta, Scipión Llona de Miraflores y María Reiche de San Isidro. Se aplicó la prueba piloto a un centro educativo de Chorrillos y a otro de Pamplona que no formaban parte de la muestra. Los resultados permitieron reformular algunos ítems de la escala de evaluación de 'cultura escolar', tomando en consideración únicamente aquellos que podían seleccionarse estadísticamente de acuerdo con los parámetros técnicos establecidos para la elaboración de escalas psicométricas.

Se solicitó el apoyo de los directores para la realización del estudio. Se tuvo acceso a la mayoría de centros educativos de los distritos elegidos para el estudio pero en muchos casos no pudo diferenciarse los tipos de cultura y se prosiguió con la preevaluación hasta lograr el propósito inicial de la investigación.

La prueba fue diseñada como una escala tipo Likert (véase anexo). En ella 
Tabla № 1

\begin{tabular}{|c|c|c|c|c|c|c|c|c|}
\hline \multicolumn{3}{|c|}{ Vida familiar } & \multicolumn{3}{|c|}{ TEST DE SCHEFFE } & \multirow[b]{2}{*}{ SLG } & \multirow[b]{2}{*}{12 Nov } & \multirow[b]{2}{*}{0} \\
\hline $\mathbf{F}$ & F Prob & Media & Grupo & Sc LI & MR & & & \\
\hline \multirow[t]{5}{*}{9.6296} & .0000 * & 1.9646 & $\mathrm{Sc} \mathrm{LI}$ & & & & & \\
\hline & & 1.9808 & MR & & & & & \\
\hline & & 2.3209 & SLG & * & & & & \\
\hline & & 2.4048 & 12 Nov & & & & & \\
\hline & & 2.5378 & $\mathrm{O}$ & * & * & & & \\
\hline
\end{tabular}

$p<0.05$

se consideraron varias áreas de acuerdo con las tareas que, según la edad de estos jóvenes, les corresponden. Para la población femenina se usaron los mismos contenidos pero los ítems fueron orientados diferenciando el género.

Las áreas finalmente constituidas para la prueba fueron:

- Área de los estudios postescuela secundaria (universitaria y técnica).

- Área de empleo.

- Área referida a la vida familiar.

Según el análisis de varianza de un factor y su comparación posthoc a través del test de Scheffe, los resultados indicaron lo que sigue.

\section{Área de promoción de la vida familiar}

En cuanto a la vida familiar (tabla $N^{\circ}$ 1) el Centro Educativo (C.E.) Ollantay la promueve más que los centros educativos Scipión Llona y María Reiche.

El C.E. San Luis Gonzaga promueve la vida familiar más que el Scipión Llona.

\section{Área de promoción de la vida laboral}

De acuerdo con el resultado del análisis (tabla $\mathrm{N}^{\circ} 2$ ) es posible afirmar que el C.E. Ollantay promueve la vida laboral más que el Scipión Llona y el María Reiche.

Por su parte, el C.E. San Luis Gonzaga promueve la vida laboral más que el Scipión Llona.

\section{Tabla № 2}

\begin{tabular}{|c|c|c|c|c|c|c|c|c|}
\hline \multicolumn{3}{|c|}{ Vida laboral } & \multicolumn{4}{|c|}{ TEST DE SCHEFFE } & \multirow[b]{2}{*}{ SL } & \multirow[b]{2}{*}{0} \\
\hline $\mathbf{F}$ & F Prob & Media & Grupo & Sc LI & MR & $12 \mathrm{Nov}$ & & \\
\hline \multirow[t]{5}{*}{13.0638} & $.0000^{*}$ & 2.2384 & Sc LI & & & & & \\
\hline & & 2.4769 & MR & & & & & \\
\hline & & 2.5714 & $12 \mathrm{Nov}$ & & & & & \\
\hline & & 2.7060 & SLG & * & & & & \\
\hline & & 2.8920 & $\mathrm{O}$ & * & * & & & \\
\hline
\end{tabular}

$p<0.05$ 
Tabla № 3

\begin{tabular}{|c|c|c|c|c|c|c|c|c|}
\hline \multicolumn{3}{|c|}{ Educación técnica } & \multicolumn{6}{|c|}{ TEST DE SCHEFFE } \\
\hline$\underline{F}$ & F Prob & Media & Grupo & Sc LI & MR & 12 Nov & SL & 0 \\
\hline \multirow[t]{5}{*}{8.0046} & $.0000^{*}$ & 2.5539 & Sc LI & & & & & \\
\hline & & 2.7147 & MR & & & & & \\
\hline & & 2.9762 & 12 nov & & & & & \\
\hline & & 3.0448 & SLG & * & & & & \\
\hline & & 3.1622 & 0 & * & * & & & \\
\hline
\end{tabular}

$p<0.05$

Área de promoción de la formación técnica

El C.E. Ollantay (tabla $\mathrm{N}^{\circ}$ 3) promueve la formación técnica con mayor énfasis que los centros educativos Scipión Llona y María Reiche.

Mientras tanto, el C.E. San Luis Gonzaga le da mayor impulso a la formación técnica que el C.E. Scipión Llona.

\section{Área de promoción de la formación universitaria}

El C.E. Ollantay (tabla $\mathrm{N}^{\circ} 4$ ) promueve la vida universitaria más que el Scipión Llona en tanto que el C.E. San Luis Gonzaga orienta más que el C.E. Scipión Llona hacia la vida universitaria.
Es importante resaltar que el patrón de promoción hacia la vida universitaria, técnica, laboral y familiar es el mismo en todos los centros educativos, pero existen diferencias significativas entre ellos.

Debido a que los centros educativos 12 de Noviembre y San Luis Gonzaga no presentan diferencias estadísticamente significativas, es posible agruparlos para incrementar el número de sujetos en el sector socioeconómico bajo.

El centro educativo que motiva más a los estudiantes a la inserción social en todas las áreas es el Ollantay de Pamplona Alta, por lo tanto, representa el tipo de cultura A.

Tabla № 4

\begin{tabular}{|c|c|c|c|c|c|c|c|c|}
\hline \multicolumn{3}{|c|}{ Educ. universitaria } & \multicolumn{6}{|c|}{ TEST DE SCHEFFE } \\
\hline $\mathbf{F}$ & F Prob & Media & Grupo & $\mathrm{Sc} \mathrm{LI}$ & MR & 12 Nov & SL & 0 \\
\hline \multirow[t]{5}{*}{5.9386} & $.0001^{*}$ & 2.8977 & Sc LI & & & & & \\
\hline & & 3.2988 & MR & & & & & \\
\hline & & 3.2799 & 12 nov & * & & & & \\
\hline & & 3.3929 & SLG & & & & & \\
\hline & & 3.4117 & 0 & * & * & & & \\
\hline
\end{tabular}

$p<0.05$ 
Los centros educativos que promueven menos hacia la inserción social en Pamplona son el 12 de Noviembre y el San Luis Gonzaga y representarían en el distrito de Pamplona la cultura B, sin embargo, debido a que al realizar el análisis se ha observado que el patrón cultural es equivalente, se ha considerado conveniente llamarlo cultura A1.

El centro educativo que promueve menos a la inserción social en todas las áreas es el Scipión Llona en Miraflores, representaría por tanto la cultura tipo B; sin embargo, debido a que la organización del patrón cultural es equivalente al de los colegios de Pamplona, se ha visto conveniente denominarlo A1 (pese a estar ubicado en otro dis- trito). La mayor orientación hacia la inserción social se presenta en el C.E. María Reiche, que ha sido considerado en función de la evaluación como representante de la cultura tipo A.

De acuerdo con lo mencionado, podemos precisar que el tipo de cultura escolar en los centros educativos evaluados, se distribuye de la siguiente manera:

\begin{tabular}{lcc}
\hline Centro educativo & $\begin{array}{c}\text { Cultura } \\
\text { A }\end{array}$ & $\begin{array}{c}\text { Cultura } \\
\text { A1 }\end{array}$ \\
\hline Ollantay & $\mathrm{X}$ & \\
12 de Noviembre & & $\mathrm{X}$ \\
San Luis Gonzaga & & $\mathrm{X}$ \\
Scipión Llona & & $\mathrm{X}$ \\
María Reiche & $\mathrm{X}$ & \\
\hline
\end{tabular}

\section{Cultura escolar}

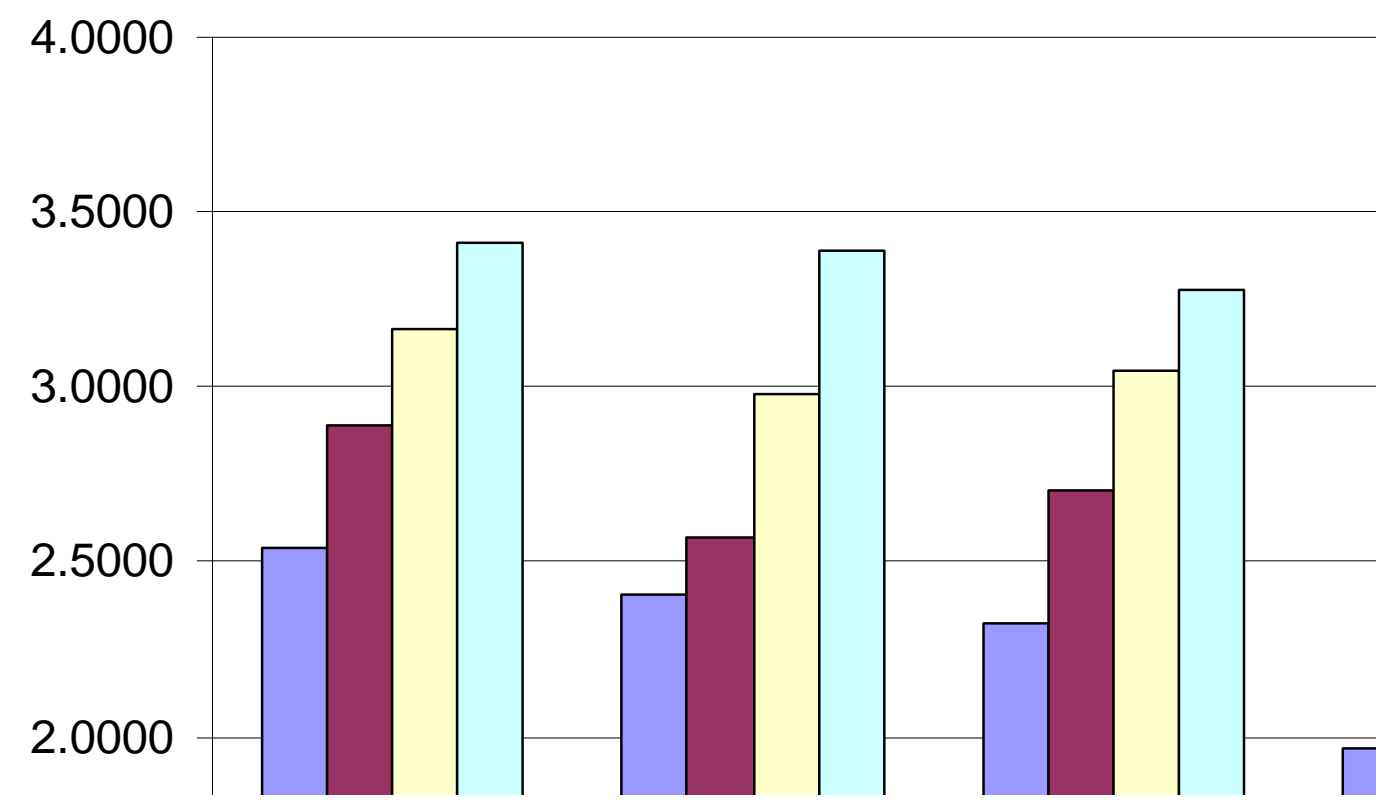


En función de los resultados obtenidos es posible señalar que se dispone de información preliminar para continuar con la realización del estudio anteriormente descrito.

\section{CONCLUSIONES}

- La cultura escolar muestra las diferencias en las que pone énfasis la escuela como organización, pudiendo a través de estas particularidades "culturales" orientar a los estudiantes de manera disímil hacia la vida laboral, académica y familiar.

- La investigación desarrollada en dos distritos de Lima ha permitido diferenciar escuelas de cultura tipo A y escuelas de cultura tipo A1. Las primeras promueven a los estudiantes con mayor intensidad hacia los ámbitos laboral, educativo (universitario y técnico) y familiar. Las de tipo A1, en cambio, lo hacen de modo menos intenso.

- El patrón de orientación hacia la inserción social es el mismo en las escuelas de cultura A y A1. Se orienta a los estudiantes, en primera instancia, a la vida universitaria, en segundo lugar hacia la educación técnica, luego hacia la actividad laboral y, finalmente, hacia la vida familiar.

- A partir de la identificación de los dos tipos de cultura escolar (A y A1), las otras variables sociales y psicológicas consideradas deben ser analizadas para determinar el senti- do e intensidad de su influencia en las estrategias de inserción social de los jóvenes estudiados.

\section{REFERENCIAS}

Ansión, J., Lazarte, A., Matos, S., Rodríguez J. \& Vega-Centeno, P. (1998). Educación: la mejor herencia. Lima: Pontificia Universidad Católica del Perú.

Delors, J. (1996). La educación encierra un tesoro. Informe a la Unesco. España: Santillana.

De Witte H. \& Lagrou, L. (1990). The quality of employment in the career of young psychologists and its impact on their job-and life satisfaction. Psychologica Belgica, 30 (1-2), 1-22. Lublin: Katholieke Universiteit Leuven.

Durkin, K. (1996). Developmental Social Psychology. Massachusetts: Blackwell Publishers.

Eurich, A. (1981). Major Transitions in the Human Cycle. D.C. Heath and Company Lexinton. Massachusetts: Lexinton Books.

Hagestad, G.O. \& Neugarten, B.L. (1985). Age and the life course. En R.H. Binstock \& E. Shanas. (Eds.), Handbook of aging and the social sciences (2a. ed.). New York: Van Nostrand Reinhold.

Instituto Nacional de Estadística e Informática. (1997). Perú: Comportamiento del empleo urbano 1995-96. Lima: INEI.

Instituto Nacional de Estadística e Informática. (1998). Perú: Caracterís- 
ticas del Empleo Urbano. Encuesta Nacional de Hogares 1997. Lima: INEI.

Jiménez, E., Lockheed, M., \& Paqueo, V. (1991). The relative efficiency of private and public schools in developing countries. The World Bank Research Observer, 6(2), 205-218.

Keren, G. (1982). Statistical and methodological issues in psychology and social sciences research. New Jersey: Hillsdale Publishers.

Kreppner K. \& Lerner, R. (Eds). (1989). Family systems and lifespan development. New Jersey: Lawrence Erlbaum Associates.

Lens, W. \& Moreas, M.A. (1994). Future time perspective: individual and societal approach. En Z. Zaleski (Ed.), Psychology of future orientation. Lublin: KUL.

Lerner, R. (1998). Adolescent development: Challenges and opportunities for research programs and policies. Annual Review of Psychology, 49, 413-46.

Maehr, M. \& Midgley, C. (1996). Transforming school cultures. Chicago: Westview Press.

Magnusson, D. (Ed.). (1996). The lifespan development of individuals. Cambridge: University Press.

Maluf, M.R. (1972). A perspectiva temporal em funcao do sexo, da idade, e do nivel sócio-economico. Revista de Psicologia Normal e Patológica, 18(3-4).

Ministerio de Educación. (1997). Nueva estructura del sistema educativo peruano. Fundamentos de la Propuesta. Lima.
Nicaise, I. (1997). Poverty and human capital. Lublin: Katholieke Universiteit Leuven, HIVA.

Nuttin, J. \& Lens, W. (1985). Future time perspective and motivation: Theory and research method. Leuven $\mathrm{y}$ Hillsdale, NJ.: Leuven University Press \& Erlbaum.

Nurmi, Jari-Erik. (1992). Age differences in adult life goals, concerns, and their temporal extension: A life course approach to future-oriented motivation. International Journal of Behavioral Development, 15(4), 487-508.

Nurmi, Jari-Erik. (1993). Adolescent development in an age-graded context: The role of personal beliefs, goals, and strategies in the tackling of developmental tasks and standards. International Journal of Behavioral Development, 16(2), 169-189.

Parodi, C. (1996). Economía y políticas sociales. Lima: Universidad del $\mathrm{Pa}$ cífico.

Pro-Joven. (1997). Informe del Proyecto y análisis preliminar de los resultados del estudio piloto. Documento sin publicar. Lima: Ministerio de Trabajo.

Psacharopoulos, G. \& Woodhall, M. (1994). Education for development. Oxford: University Press, Inc.

Rebok, G. (1987). Lifespan cognitive development. New York: Holt, Rinehart \& Winston.

Schwebel, M., Maher, C., \& Fagley, N. (Eds.). (1990). Promoting cognitive growth over the lifespan. Hillsdale, New Jersey: Lawrence Erlbaum Associates, Publishers. 
Trommsdorff, G. (1983). Future orientation and socialization. International Journal of Psychology, 18, 381-406.

UNICEF \& Cuánto. (1997). Reporte acerca del desempleo y subempleo en el Perú. Documento sin publicar. Lima.

Van Der Keilen. (1982). Extension of future time perspective and attitude towards present, past and future in normal and socially handicapped adolescents. Influence of experimentallyinduced success and failure. Psychologica, 22(2), 161-183.

Vondracek F., Lerner, R., \& Schulenberg, J. (1986). Career development. New Jersey: Lawrence Erlbaum Associates.

Zaleski, Z. (Ed.). (1994). Psychology of future orientation. Lublin: KUL, Towarzystwo Naukowe. 


\section{Anexo \\ Evaluación de cultura escolar}

En las siguientes páginas Ud. encontrará un conjunto de afirmaciones acerca de su colegio. Tenemos interés de conocer lo que piensa de cada una de ellas y si le parece que son aplicables o no a su centro educativo. Por favor, exprese su opinión para cada una de las afirmaciones colocando un círculo en los números que aparecen en la hoja de respuestas tal como se indica en el presente ejemplo:

\section{Nuestro colegio participa en competencias deportivas}

\begin{tabular}{ccccc}
\hline Aplicable & $\begin{array}{c}\text { De alguna manera } \\
\text { aplicable }\end{array}$ & $\begin{array}{c}\text { No es aplicable } \\
\text { ni inaplicable }\end{array}$ & $\begin{array}{c}\text { De alguna manera } \\
\text { inaplicable }\end{array}$ & Inaplicable \\
\hline 5 & 4 & 3 & 2 & 1 \\
\hline
\end{tabular}

Si Ud. considera que su colegio promueve la participación en eventos deportivos e incentiva de manera constante a los estudiantes y profesores a realizar actividades deportivas deberá poner un círculo en el número 5. Si su centro educativo participa en competencias deportivas pero no realiza un gran esfuerzo por generar un clima deportivo en la comunidad educativa, Ud. marcará 4. Si su respuesta es neutra deberá marcar el 3. Si Ud. conoce que su colegio puede participar en competencias deportivas pero observa que no existe mayor interés en asistir a ellas y si es posible se evita que el centro participe, entonces deberá marcar 2 y si no se participa en competencias deportivas marcará 1 .

No existen respuestas correctas o incorrectas, sólo su opinión sincera y personal es de interés para nosotros. Las respuestas que Ud. emita serán anónimas y los resultados se tratarán de manera confidencial. Por favor, sea honesto en sus respuestas, ninguno de los miembros de su centro educativo podrá acceder a ellas de manera personal.

Muchas gracias por su colaboración.

1. En nuestro colegio se pone énfasis en que es necesario trabajar para poder estudiar en una institución técnica de manera paralela.

2. En nuestro centro educativo los docentes motivan a los escolares para que al terminar la secundaria inicien sus estudios universitarios.

3. En nuestro centro educativo los docentes motivan a los escolares para que al terminar la secundaria inicien estudios en centros educativos de nivel técnico.

4. En nuestro centro educativo se enfatiza en que para financiar los estudios técnicos es necesario trabajar primero.

5. En nuestro centro educativo se promueve la participación de las diferentes universidades para que brinden información a los educandos con respecto a las diferentes facultades existentes en ellas.

6. En nuestro centro educativo los docentes motivan a los chicos para que al terminar la secundaria apoyen a su familia en las labores hogareñas diarias.

7. En nuestro centro educativo los docentes motivan a los escolares para que al terminar la secundaria inicien inmediatamente actividades laborales y no continúen estudiando.

8. En nuestro centro educativo se promueve la participación de los diferentes centros de capacitación técnica para que brinden información a los educandos con respecto a las diferentes espe- 
cialidades existentes en ellas.

9. En nuestro centro educativo se orienta considerablemente a los chicos a encargarse de su familia antes que continuar sus estudios o trabajar.

10. En nuestro centro educativo se brinda información con respecto a las oportunidades de trabajo existentes en nuestro medio.

11. En nuestro colegio existe la posibilidad de recibir orientación vocacional con respecto a carreras universitarias.

12. En nuestro colegio existe la posibilidad de recibir orientación vocacional con respecto a carreras técnicas.

13. En nuestro colegio existe la posibilidad de recibir asesoría con respecto a sus posibilidades laborales de acuerdo a sus habilidades.

14. A lo largo de todo el proceso educativo Ud. ha sido estimulado de manera constante para lograr un título universitario.

15. En el colegio existe la posibilidad de recibir orientación para enfrentar en mejores condiciones nuestra vida familiar apenas se concluyan los estudios secundarios.

16. A lo largo de todo el proceso educativo Ud. ha sido estimulado de manera constante para que al terminar sus estudios secundarios consiga inmediatamente un trabajo.

17. Durante su formación escolar se ha remarcado el hecho de que un profesional enfrenta los retos de la vida en mejores condiciones que un técnico.

18. A lo largo de todo el proceso educativo Ud. ha sido estimulado de manera constante para lograr un título técnico.

19. Durante la formación escolar se ha remarcado el hecho de que los chicos deberían colaborar en las labores familiares cotidianas antes que estudiar o trabajar

20. Durante su formación escolar se ha remarcado el hecho de que una persona que dispone de un trabajo enfrenta mejor los retos de la vida que alguien que decide continuar estudiando.

21. En el colegio se ha difundido de manera continua información referente a las posibilidades de continuar estudios universitarios.

22. Durante su formación escolar se ha remarcado el hecho de que un técnico enfrenta los retos de la vida en mejores condiciones que un profesional universitario.

23. En el colegio se ha difundido de manera continua información referente a las posibilidades laborales existentes en el mercado con la finalidad de ayudar a los estudiantes a conseguir empleo inmediatamente.

24. En nuestro colegio se pone énfasis en que es necesario trabajar para poder estudiar en la universidad de manera paralela.

25. En nuestro centro educativo se considera que un chico no ha sido exitoso en su vida si prefiere ayudar a sus padres antes de trabajar o continuar con sus estudios.

26. En el centro educativo se brinda entrenamiento técnico a los escolares para que al terminar la secundaria se encuentren capacitados para conseguir un empleo inmediatamente.

27. En nuestro centro educativo los profesores enfatizan en que para financiar los estudios universitarios es necesario trabajar primero.

28. En nuestro centro educativo se motiva a las chicas a formar su propia familia apenas terminen de estudiar la secundaria.

29. En nuestro colegio se estimula constantemente a los estudiantes a que trabajen de manera paralela a los estudios.

30. En el colegio se ha difundido de manera continua información referente a posibilidades de continuar estudios técnicos. 